\title{
Power Management of Monolithic 3D Manycore Chips with Inter-tier Process Variations
}

\author{
ANWESHA CHATTERJEE and SHOUVIK MUSAVVIR, Washington State University, \\ Pullman, Washington, USA \\ RYAN GARY KIM, Colorado State University, Fort Collins, Colorado, USA \\ JANARDHAN RAO DOPPA and PARTHA PRATIM PANDE, Washington State University, \\ Pullman, Washington, USA
}

\begin{abstract}
Voltage/frequency island (VFI)-based power management is a popular methodology for designing energyefficient manycore architectures without incurring significant performance overhead. However, monolithic 3D (M3D) integration has emerged as an enabling technology to design high-performance and energyefficient circuits and systems. The smaller dimension of vertical monolithic inter-tier vias (MIVs) lowers effective wirelength and allows high integration density. However, sequential fabrication of M3D layers introduces inter-tier process variations that affect the performance of transistors and interconnects in different layers. Therefore, VFI-based power management in M3D manycore systems requires the consideration of inter-tier process variation effects. In this work, we present the design of an imitation learning (IL)-enabled VFI-based power-management strategy that considers the inter-tier process-variation effects in M3D manycore chips. We demonstrate that the IL-based power-management strategy can be fine-tuned based on the M3D characteristics. Our policy generates suitable V/F levels based on the computation and communication characteristics of the system for both process-oblivious and process-aware configurations. We show that the proposed process-variation-aware IL-based VFI implementation for M3D manycore chips lowers the overall energy-delay-product (EDP) by up to $16.2 \%$ on average compared to an ideal M3D system with no M3D process variations.
\end{abstract}

CCS Concepts: $\bullet$ Hardware $\rightarrow$ 3D integrated circuits; $\bullet$ Computer systems organization $\rightarrow$ Multicore architectures; $\bullet$ Hardware $\rightarrow$ Chip-level power issues; $\bullet$ Computing methodologies $\rightarrow$ Supervised learning; $\bullet$ Hardware $\rightarrow$ System on a chip;

Additional Key Words and Phrases: Monolithic 3D, MIV, imitation learning, DVFI, inter-tier process variation, EDP

\section{ACM Reference format:}

Anwesha Chatterjee, Shouvik Musavvir, Ryan Gary Kim, Janardhan Rao Doppa, and Partha Pratim Pande. 2020. Power Management of Monolithic 3D Manycore Chips with Inter-tier Process Variations. J. Emerg. Technol. Comput. Syst. 17, 2, Article 13 (December 2020), 19 pages.

https://doi.org/10.1145/3430765

This work was supported, in part by the US National Science Foundation (NSF) grants CNS-1955353, CNS-1564014 and USA Army Research Office grant W911NF-17-1-0485.

Authors' addresses: A. Chatterjee, S. Musavvir, J. R. Doppa, and P. P. Pande, 355 NE Spokane St, Pullman, WA 99163; emails: \{anwesha.chatterjee, shouvik.musavvir\}@wsu.edu; R. G. Kim, 1373 Campus Delivery, Fort Collins, CO, 80524; email: ryan.g.kim@colostate.edu.

Permission to make digital or hard copies of all or part of this work for personal or classroom use is granted without fee provided that copies are not made or distributed for profit or commercial advantage and that copies bear this notice and the full citation on the first page. Copyrights for components of this work owned by others than ACM must be honored. Abstracting with credit is permitted. To copy otherwise, or republish, to post on servers or to redistribute to lists, requires prior specific permission and/or a fee. Request permissions from permissions@acm.org.

(C) 2020 Association for Computing Machinery.

1550-4832/2020/12-ART13 \$15.00

https://doi.org/10.1145/3430765 


\section{INTRODUCTION}

Three-dimensional (3D) integration has emerged as a promising paradigm to design highperformance and energy-efficient manycore chips [1, 2, 41, 43]. Through-silicon-via (TSV) technology has been the prevailing technology used to integrate multiple planar dies into a single 3D design [3, 42, 44, 45]. However, TSV-based 3D integration has well-known limitations such as high area overhead due to large keep-out-zones (KOZ) around TSVs, electromigration-induced stress, and thermal hot spots [3, 4, 32, 38]. Recently, monolithic 3D (M3D) integration has been introduced as an alternative to TSV-based 3D technology. In M3D, multiple tiers are processed sequentially on the same die and use monolithic inter-tier vias (MIVs) as the vertical interconnects $[5,6]$. Compared to TSVs, MIVs are significantly smaller and allow lower effective wire length, higher integration density, and enhanced energy-efficiency [5, 7, 8, 31].

Combining 3D integration with networks-on-chip (NoCs) provides a scalable, highperformance, and energy-efficient communication backbone for manycore systems. In an M3Denabled manycore chip, processors, NoC routers, and NoC links can take advantage of the smaller MIVs to create true 3D circuits across multiple tiers [9]. However, state-of-the-art M3D integration faces technology- and fabrication-related challenges that negatively impact the performance of M3D circuits. The sequential integration of M3D requires a low-temperature top-tier annealing process to prevent any degradation in bottom-tier transistors [10,22] and alternative metals (e.g., tungsten) for the bottom-tier interconnects to withstand the high fabrication temperatures [11, 22]. However, these decisions slow down the top-tier transistors and bottom-tier interconnects resulting in inter-tier process variations. The effects of this inter-tier process variation directly impact the performance of the M3D processors and the NoC. To mitigate these effects, the design of a process-variation-aware M3D processor has been proposed [12].

In addition to the inter-tier process-variation concerns, the power density of the M3D system increases over its 2D counterpart due to its smaller footprint. Hence, to complement the performance advantage due to $\mathrm{M} 3 \mathrm{D}$, we need to also incorporate efficient power-management strategies to manage energy and thermals. Voltage-frequency islands (VFI) is a popular power-management methodology that is applied to manycore chips [26,46]. VFIs have been demonstrated to be an important tool to improve the energy-efficiency and the thermal profile of M3D-based manycore designs [13]. However, state-of-the-art VFI-based power-management strategies for M3D systems are oblivious to the process-variation-induced performance degradations. Therefore, it is important to study and characterize the effects of process variations on M3D power-management strategies.

In this work, for the first time, we study the impacts of process variations on powermanagement strategies for M3D-based manycore chips. We propose an imitation-learning-based M3D power-management strategy that assigns voltage and frequency $(\mathrm{V} / \mathrm{F})$ levels to cores and $\mathrm{NoC}$ elements to minimize the effects of inter-tier process variations. We demonstrate that this power-management strategy can account for the M3D process variations and be fine-tuned even for a process-variation-unaware M3D system design. Furthermore, we show that the process-unaware DVFI implementation leads to suboptimal performance in an M3D manycore system. For both process-variation-aware and -unaware M3D configurations considered in this work, VFI-based power management is capable of saving energy by more than $20 \%$ compared to an ideal M3D system, without introducing significant performance overhead.

\section{RELATED WORK}

Existing works have explored the advantages of M3D-based circuits and systems [14, 15]. M3Dbased designs show significant reduction in chip area, total wirelengths, and improved energy efficiency compared to their planar counterparts $[16,18]$. For example, for an LDPC (low density 
parity check) benchmark circuit, the footprint and wirelength of planar designs are reduced by $44 \%$ and 34\%, respectively, by using M3D in the $45 \mathrm{~nm}$ technology node. This results in a $9 \%$ reduction of the longest path delay and a $32 \%$ reduction in the total power consumption [17]. Additionally, it was demonstrated that the performance improvement increases with smaller technology nodes [18-20]. The effects of the number of planar tiers, tier-level partitioning, and MIV insertion methodology on the performance and power consumption of M3D-based ICs were analyzed in Reference [21]. It was also demonstrated that increasing the number of MIVs inserted in the design improves the power savings in M3D-based ICs. The authors in Reference [9] proposed the design of M3D-enabled energy-efficient NoC architectures. The M3D-enabled NoC, on average, achieves 28\% lower energy-delay-product (EDP) than its TSV-based counterpart. However, the works discussed above do not consider the fabrication-related challenges in M3D integration.

Several works have explored the challenges associated with the M3D fabrication and manufacturing processes $[11,22]$. Due to high temperatures, sequential fabrication of the top tier over the bottom tier affects the quality of transistors and interconnects in the bottom tier during the ion implantation and thermal annealing processes. Two techniques have been proposed to realize top-tier transistors without damaging the components in the lower tiers: (i) a low-temperature fabrication process $\left(500-650{ }^{\circ} \mathrm{C}\right.$ [11]) in the top tier using solid phase epitaxy regrowth (SPER) [23] or laser annealing [24]; and (ii) using tungsten as an alternative interconnect in the bottom tier [22]. For the first case, transistors in the top tiers have significant deviation from the nominal source-drain current value [11]. For the second case, tungsten can withstand the high temperature process, but the higher resistivity in tungsten leads to inferior performance of the bottom-tier interconnects [22].

There are few works that explore the process variation effects on a realistic M3D design. In Reference [12], the authors found that the energy consumption and delay are increased by about $30 \%$ and $17 \%$, respectively, compared to an ideal M3D process. In Reference [12], authors proposed a process-variation-aware design methodology for M3D NoCs. Recently, a process-variation-aware design of a processing core in M3D has been demonstrated [25]. It is to be noted in this work, that even with process variation, an M3D processor outperforms its TSV-based counterpart. Expectedly, this work shows that the reduced wire length and area result in higher operating frequency, which in turn increases the power density of an M3D system significantly. Hence, an M3D-based manycore system will be benefited by incorporating suitable power-management strategies.

Power management in M3D-based manycore systems has been explored recently in Reference [13]. This work shows that an M3D-based system with power management achieves significant performance improvements with much lower EDP than its TSV-based counterpart. It is also seen that the difference between the maximum and minimum temperatures in an M3D manycore system is insignificant [13]. However, the work presented in Reference [13] does not consider the effects of process variation or multi-tier processors enabled by M3D. Outside of M3D-based systems, dynamic voltage frequency islands (DVFI) is a very popular methodology to efficiently reduce the energy of a manycore system without significantly compromising the performance [26-28]. Machine learning techniques for power management in manycore systems have been thoroughly investigated [29]. Recent work shows that designing an imitation learning (IL)-based DVFI outperforms other popular DVFI-based power-management techniques, such as reinforcement learning (RL) and traditional feedback control [30]. RL techniques require large amount of training data and their time to convergence is very high due to the need to explore huge number of state-action pairs to reach the solution. Compared to RL, IL-based techniques are light-weight solutions that require less data and time to reach near-optimal solutions [30,47]. Therefore, in this work, we adopt an IL-based approach to implement DVFI on process-degraded M3D systems. 
Table 1. M3D Architectures with their Nominal Frequencies for a $28 \mathrm{~nm}$ Technology Node

\begin{tabular}{c|c|c|c}
\hline M3D Architecture & Design Consideration & Evaluation Consideration & Nominal Frequency \\
\hline Iso & No Process Variation & No Process Variation & $3.50 \mathrm{GHz}$ \\
\hline Naïve & No Process Variation & Process Variation & $3.16 \mathrm{GHz}$ \\
\hline Hetero & Process Variation & Process Variation & $3.44 \mathrm{GHz}$ \\
\hline
\end{tabular}

In this work, our aim is to show that IL-based DVFI can learn the M3D inter-tier process variation effects on the system and determine the best V/F of each VFI cluster. We will demonstrate that this improves the power-performance tradeoff of the whole system.

\section{M3D MANYCORE SYSTEM DESIGN}

In this section, we discuss the design space, advantages, and challenges of an M3D-based manycore chip that consists of cores and the NoC (routers and links). We first present the salient features of an M3D core followed by the characteristics of the NoC.

When designing a multi-tier M3D core, we can focus on reducing the interconnect lengths in critical paths of the design and increase the overall clock speed. However, like many other works [14-21], if we do not consider the process variation effects, the critical path could face unexpected slowdowns and the achievable frequency of the system could be affected. Therefore, in this work, we consider three different core architectures under different design scenarios as demonstrated in Reference [25]. Iso represents the ideal M3D architecture and both design and performance evaluation of Iso does not consider M3D process variations in the system. Although Iso is an unrealistic scenario given current M3D fabrication processes [22-24], we present these results to demonstrate the upper-bound on performance. In the Naïve M3D architecture, no process variation is considered at the design stage. However, in the evaluation phase, we considered M3D process variations. Hetero considers the process variation effects during design and evaluation of the M3D system. This process-variation-aware case mitigates their effects through effective M3D core design [25]. These M3D core configurations are listed in Table 1 with the design assumptions, evaluation assumptions, and their frequency at $1 \mathrm{~V}$ nominal voltage for the $28 \mathrm{~nm}$ technology node. Since Iso considered no process variations during evaluation, there is no degradation on the performance of the critical paths. This Iso M3D core is shown to be around $17 \%$ faster in frequency than the planar cores [25]. For traditional planar circuits, the nominal frequency is $3 \mathrm{GHz}$ for a $28 \mathrm{~nm}$ technology node at $1 \mathrm{~V}$ nominal voltage following ITRS [42]. Hence, the Iso frequency is set at $3.5 \mathrm{GHz}$ as mentioned in Table 1 . However, since M3D processes have significant inter-tier variations, the Naïve M3D core demonstrates realistic performance with only around $6 \%$ faster than the planar cores in terms of frequency [25]. In Hetero, the cores are designed to place critical path components in the lower tier to avoid the transistor degradation in the top tier. This asymmetric partitioning of structures results in $15 \%$ faster cycles compared to a planar core [25]. The corresponding frequencies for Naïve and Hetero are listed in Table 1. We will study the effectiveness of the IL-based power-management strategy by considering each of these three processor architectures in the manycore system.

In a manycore architecture, the NoC works as the communication backbone and affects the overall system execution time and energy consumption. Hence, optimal placement of cores, routers, and inter-router links is necessary to achieve low network latency and reduced energy consumption. However, considering the challenges associated with M3D fabrication, designing an $\mathrm{NoC}$ in $\mathrm{M} 3 \mathrm{D}$ technology requires the consideration of inter-tier process variation effects on the routers and links. The inter-tier process degradations are manifested in a slowdown of bottom-tier interconnects and top-tier transistors in a router. We have recently demonstrated that without 


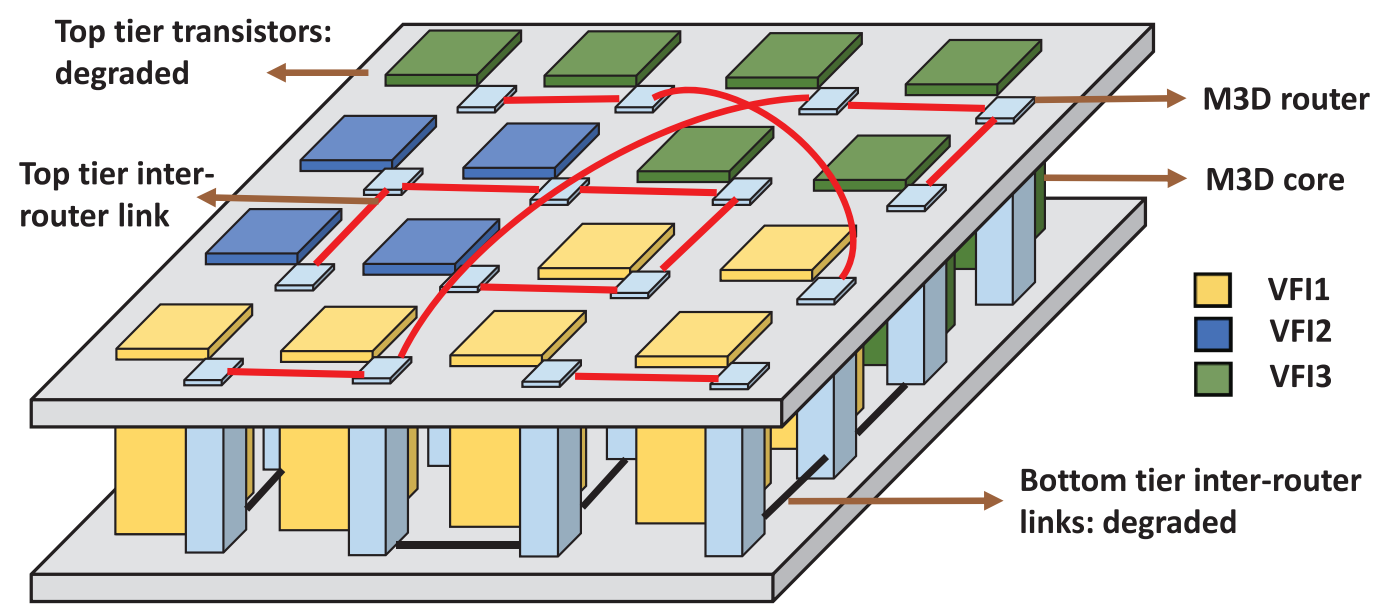

Fig. 1. A VFI-enabled 16-core two-tier M3D system with inter-tier process variation. Here, the system is divided into three VFIs as an example. The associated NoC router is also included in that VFI.

considering inter-tier process variation, an M3D-enabled NoC architecture overestimates the achievable performance significantly [12]. The NoC design and optimization method should distribute the intra-router stages and inter-router links among the tiers to mitigate the adverse effects of process variation. In this work, a small-world NoC is used, since it outperforms other regular or irregular NoC architectures [39]. To ensure that we do not introduce additional delays through VFIs, we introduce an additional constraint into the NoC optimization that guarantees that all cores in a VFI make a connected graph using only intra-VFI links (VFI creation is discussed in detail in Section 4.1). Like the M3D cores, the NoC design for Iso architecture has no process variationrelated delays. The NoC design for Naïve architecture has the same Iso configuration where the process degradation in the routers and links is considered during the performance evaluation only. The NoC design of the Hetero architecture includes router delays and inter-router link delays due to inter-tier process variations according to the design methodology proposed in Reference [12].

An example of a VFI-enabled M3D manycore chip is shown in Figure 1. The figure shows a 16core two-tier M3D system, divided into three VFIs with the distribution of inter-router links in top and bottom tier of the M3D chip based on the design of the small-world NoC from Reference [12].

\section{POWER MANAGEMENT ON M3D SYSTEMS}

As manycore platforms are becoming ubiquitous, it is imperative to enhance their energy efficiency without sacrificing noticeable performance. VFI-based power management is an effective methodology to make manycore chips energy-efficient without giving rise to significant performance penalties. In a VFI-based manycore chip, we divide the whole system into multiple clusters based on the workload and assign a V/F to each cluster. As we have discussed in Section 2, an ILbased VFI framework is more efficient than any other existing methodologies [30]. In this section, we provide an overview of the IL-based VFI framework and how we can leverage workload and traffic characteristics of an M3D system with different kinds of core architectures (i.e., Iso, Naïve, and Hetero).

\subsection{VFI Power Management Policy}

Before we discuss how to implement a DVFI policy on M3D-based systems, we will first discuss DVFI policies for manycore systems in general. To implement a DVFI policy, the first step is to create clusters (islands) of cores and routers where all cores and their associated routers and links 
have similar behavior and can benefit from V/F tuning. We consider the instruction per cycle (IPC) and the inter-core traffic pattern to create the VFIs. We use the $k$-means clustering algorithm and apply the hybrid clustering proposed in Reference [28] to minimize the intra-cluster variation of both IPC and communication. Hybrid clustering involves first creating core clusters with similar IPC characteristics, followed by an additional clustering of cores and routers for each IPC cluster based on their traffic patterns. The clusters obtained are physically fixed in the system and we dynamically tune the V/F of each cluster.

Our next step is to create an efficient controller to tune the V/F levels of each cluster. We use IL to learn a good policy for dynamically tuning the V/F levels of the VFI clusters. IL is a supervised learning methodology that creates a policy that learns from numerous observations given by an expert or an Oracle as the solution to a sequential design process. Essentially, the Oracle demonstrates to the learner how to tune the V/F levels of each VFI based on the state of the cluster to minimize the overall EDP of the system. Therefore, to learn a good policy, we require a suitable Oracle to guide the learning process and follow the process in Reference [30] to generate the Oracle.

Let us consider an application divided into a total of $N$ control epochs and $M$ VFIs. For each epoch $t$, the DVFI policy $\pi$ takes the current state of the system, $s_{t}$, and allocates a V/F value to each of the VFIs:

$$
\pi: s_{t} \rightarrow\left\{V_{1} / F_{1}, V_{2} / F_{2}, \ldots, V_{M} / F_{M}\right\}
$$

The state $s_{t}$ should capture the system characteristics at epoch $t$ that reflects on the system performance and energy consumption. The core computation and inter-node communication patterns at $t$ as well as the last seen $\mathrm{V} / \mathrm{F}$ level can be correlated to the resulting power and performance of the system. Therefore, we define the state $s_{t}$ of the system at epoch $t$ as:

$$
s_{t}:\left\{i p c, f_{i j}, \pi\left(s_{t-1}\right)\right\}
$$

where ipc is IPC characteristics, $f_{i j}$ is the traffic pattern between each ith source core to $j$ th destination core and $\pi\left(s_{t-1}\right)$ represents the previous state $\mathrm{V} / \mathrm{F}$ level of the system.

The total energy consumed by the system in this process is given by:

$$
E(\pi)=\sum_{t=1}^{N} \epsilon\left(t, \pi\left(s_{t}\right)\right),
$$

where $\epsilon\left(t, \pi\left(s_{t}\right)\right)$ represents the energy consumed at epoch $t$ for $\mathrm{V} / \mathrm{F}$ allocation of $\pi\left(s_{t}\right)$. Similarly, the total execution time of the system is defined as:

$$
T(\pi)=\sum_{t=1}^{N} \tau\left(t, \pi\left(s_{t}\right)\right),
$$

where $\tau\left(t, \pi\left(s_{t}\right)\right)$ represents the execution time at epoch $t$ for $\mathrm{V} / \mathrm{F}$ allocation of $\pi\left(s_{t}\right)$. The total EDP of the system under policy $\pi$ represents the overall performance of the system, which is defined as:

$$
\operatorname{EDP}(\pi)=E(\pi) * T(\pi)
$$

To create a good Oracle, our objective in a DVFI process is to minimize the energy dissipation of the system within a specified performance penalty, $p$ :

$$
\begin{aligned}
& \pi^{*}=\operatorname{argmin}_{\pi} \operatorname{EDP}(\pi) \\
& \text { s.t. } \frac{T(\pi)}{T\left(\pi_{\text {nom }}\right)} \leq p
\end{aligned}
$$

where $\pi^{*}$ is the Oracle policy and $\pi_{n o m}$ is the policy that always uses the nominal V/F of the system. The Oracle creation can be done offline by characterizing the application running on the 


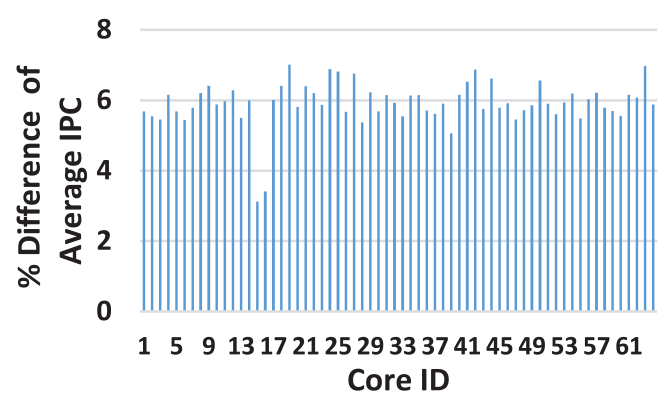

(a)

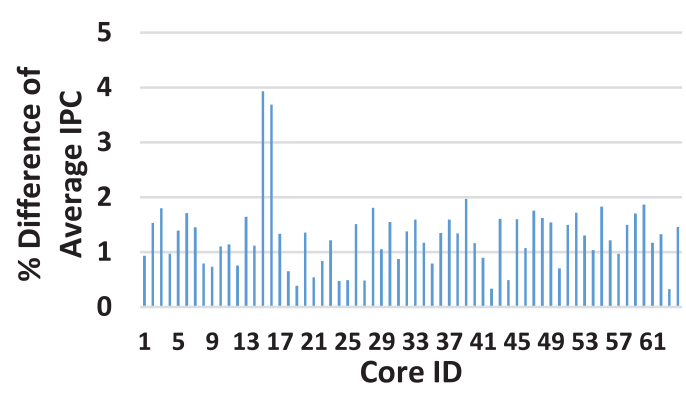

(b)

Fig. 2. Percentage difference in average core IPC for (a) Naïve and (b) Hetero with respect to Iso for a 64-core system running FFT.

M3D manycore system at different V/F levels and perform a search to get the optimal joint V/F allocations for all the VFIs. As the number of control epochs increases, the computation time to search for the global optimum increases exponentially. Therefore, at each epoch, we perform a local optimization to find the local optimal $M \mathrm{~V} / \mathrm{F}$ levels (one for each VFI) that minimizes the EDP at each epoch for $x$ different time penalties (0\%,5\%, and $10 \%$ in our case for $x=3$ [30]). This process gives us $x$ different sets of $\mathrm{V} / \mathrm{Fs}$ at each epoch. Then, we split the $N$ application control epochs into sets of $y$ consecutive disjoint control epochs. For each of these smaller sets of $y$ epochs, we choose the set of $\mathrm{V} / \mathrm{F}$ levels at each epoch (one of the $x$ found previously) that minimizes the EDP within the performance penalty $p$ across the consecutive $y$ epochs. All of these chosen V/F levels constitute the Oracle policy $\pi^{*}$. The quality of the Oracle is indicated by how much overall EDP we can minimize for the system. It is seen to saturate at $y=15$ [30]. This allows us to scale to larger applications without significant loss of fidelity in the Oracle.

Given this Oracle policy, the goal of the IL-based VFI policy $\pi$ is to imitate (learn) the Oracle policy $\pi^{*}$ and predict V/F levels for each VFI, such that the learner minimizes: $E D P(\pi)-E D P\left(\pi^{*}\right)$. This can be achieved with any regression-based learner such as linear regression or regression tree. However, a standard regression learner considers the input examples independently distributed. For a DVFI-based system, state $s_{t}$ is dependent on the previous state $s_{t-1}$. Therefore, standard supervised learning may lead to error propagation in the learner and lead to more errors. Therefore, considering our DVFI problem as a structure prediction problem, we apply the DAgger algorithm [33], which generates additional training data when predictions are wrong to learn from its mistakes.

\subsection{VFI on M3D Architectures}

Based on our discussion in Section 3, M3D system performance gets affected by the processor degradations due to the inter-tier process variation effects. For example, the M3D inter-tier process variation can slowdown the critical paths, increase the overall clock period, and thereby affect the IPC. Since each M3D system operates at a different frequency, the computation and communication operations are not carried out at the same rate for the Iso, Naïve, and Hetero M3D systems. This also has a non-uniform effect across the cores, since this will more greatly affect tasks that have to access main memory more often. This will ultimately affect the IPC of each core, since the memory stall times will be affected. This has two major effects on our VFI-based power-management policy for M3D systems. First, since the inputs to our policy include IPC, this can cause our policy to see a different system state and make a different V/F decision. Second, these different M3D characteristics can affect the power-performance tradeoffs and invalidate the policy we had learned using the ideal M3D system. This similarly applies to the traffic in the system. Figure 2 shows the 


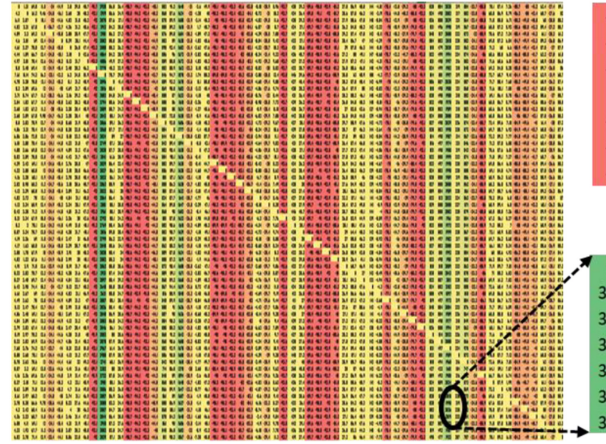

(a)

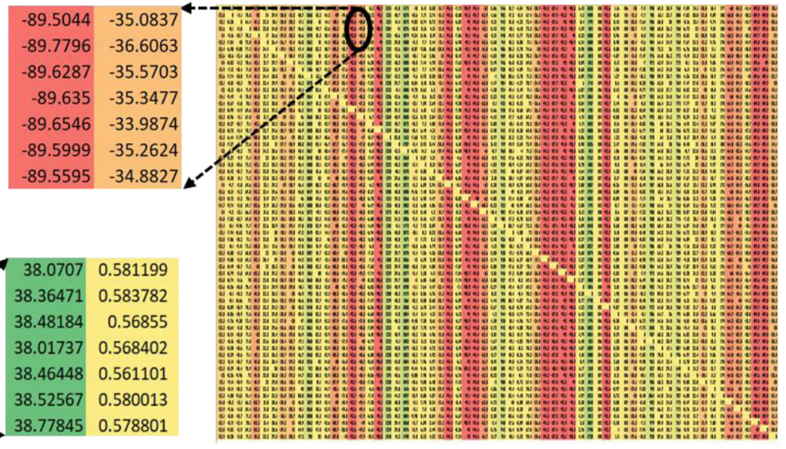

(b)

Fig. 3. Percentage difference in overall traffic rate of each source (row) to destination (column) core in (a) Naïve and (b) Hetero with respect to Iso for a 64-core system running FFT. Red cells refer to decrease in traffic while green cells correspond to an increase in traffic.

difference in average IPC of each core considering the Naïve and Hetero architectures with respect to its Iso counterpart for a 64-core system running FFT. The average change in IPC is $5.9 \%$ and $1.3 \%$ for Naïve and Hetero, respectively. This clearly shows that the process variations have a noticeable effect on the IPC.

Similarly, Figure 3 shows the difference in overall traffic between each source core (row) to destination core (column) for the Naïve (Figure 3(a)) and Hetero (Figure 3(b)) architectures with respect to Iso for a 64-core system running FFT as an example. The red cells represent a decrease in traffic and the green cells represent an increase in traffic compared to the Iso system. The average change in traffic is $32.5 \%$ and $28.9 \%$ for Naive and Hetero, respectively.

These IPC and traffic characteristics are influenced by the specific M3D core architecture. Therefore, the VFI policy needs to capture the varied characteristics of the input state. The input parameters for a learner policy is the current state of the system $s_{t}$ as shown in Equation (2), where $s_{t}$ is represented by the IPC and the traffic patterns $\left(f_{i j}\right)$ at epoch $t$ as follows:

$$
s_{t}:\left\{i p c_{M 3 D}, f_{i j_{M 3 D}}, \pi_{M 3 D}\left(s_{t-1}\right)\right\}
$$

where $i p c_{M 3 D}$ and $f_{i j_{M 3 D}}$ capture the effects of inter-tier process variation for different M3D architectures and $\pi_{M 3 D}\left(s_{t-1}\right)$ represents the $\mathrm{V} / \mathrm{F}$ allocation taken up in the previous state of the M3D system. Our power-management policy needs to learn the new mapping of IPC and traffic characteristics to V/F for a specific M3D architecture for efficient V/F tuning. Therefore, the VFI policy for the Naïve M3D architecture can be modified from Equations (1) and (2) as:

$$
\begin{aligned}
\pi_{\text {native }}: S_{t} \rightarrow\left\{V_{1} / F_{1}, / V_{2} / F_{2}, \ldots, V_{M} / F_{M}\right\}_{\text {native }} \\
\text { where } S_{t}:\left\{i p c_{\text {naive }}, f_{i j_{\text {naive }}}, \pi_{\text {naive }}\left(s_{t-1}\right)\right\}
\end{aligned}
$$

Thus, we apply Equations (1) to (6) to create the Oracle policy for Naïve and use the subsequent IL-learning algorithms to create the policy $\pi_{\text {naive }}$ that properly tunes the V/F for Naïve. Similarly, a DVFI policy $\pi_{\text {hetero }}$ is designed to dynamically tune the V/F levels of the Hetero M3D architecture using the specific Hetero IPC and traffic characteristics.

The overall flow of the IL-based DVFI implementation is described in Figure 4. The top block obtains the M3D-architecture-specific IPC and $f_{i j}$ metrics and uses those metrics to create the VFI clusters and core, routers, and link placements (Section 3). The middle block then deploys this VFIpartitioned M3D system design on a full-system simulator and collects traces of IPC, $f_{i j}$, power, execution time, and so on, at each epoch to formulate the Oracle policy and perform the offline 


\section{M3D System Characterization and Optimization (Section 3)}

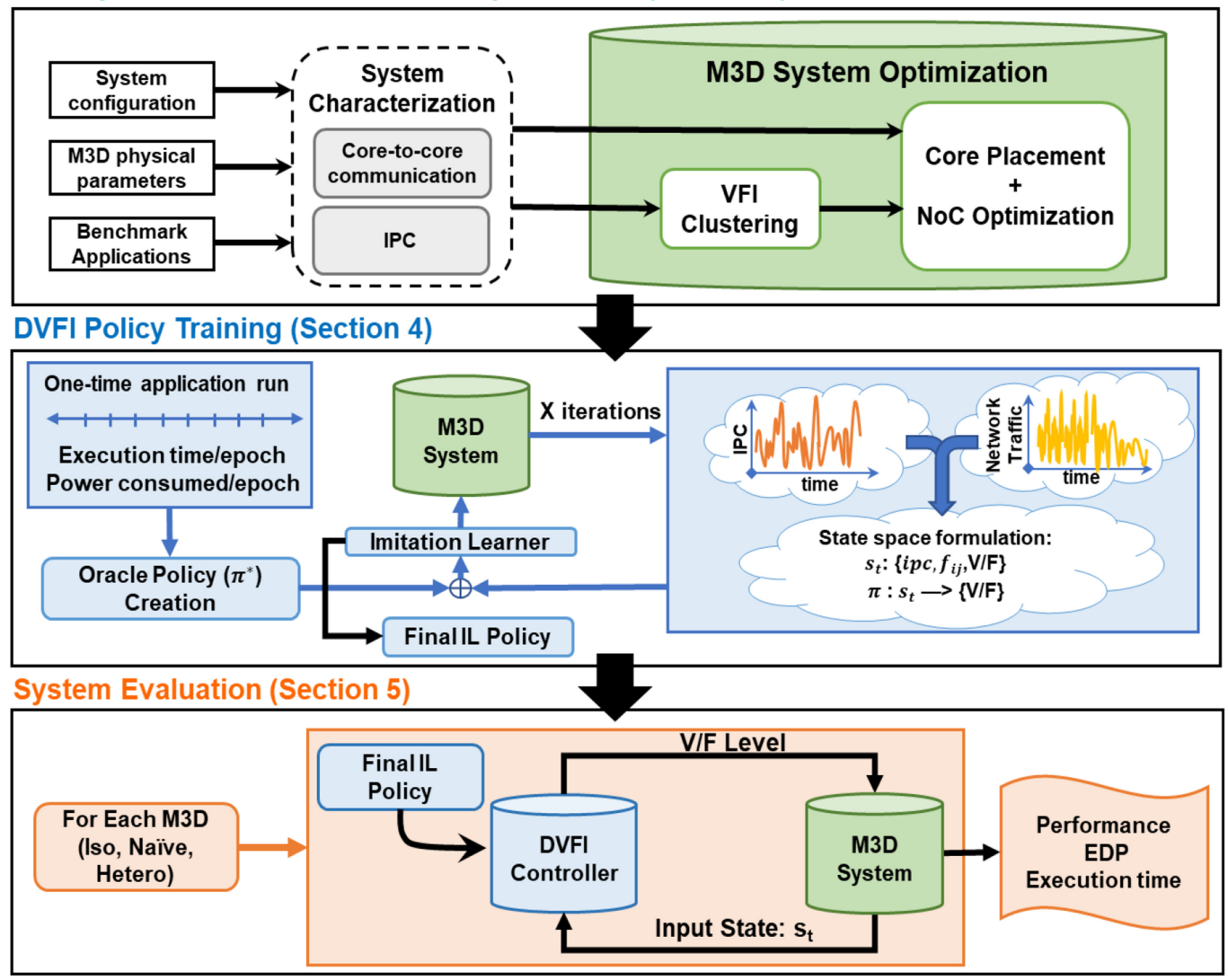

Fig. 4. The overall flow of the M3D process-variation-aware IL-based DVFI implementation for M3D manycore systems.

training of the IL-based DVFI learner (Section 4). The bottom block evaluates the IL-based DVFI policy on the M3D manycore system (Section 5).

\section{RESULTS AND ANALYSIS}

In this section, we describe the M3D manycore system on which we apply our DVFI control technique. Then, we analyze the effectiveness of the IL policy for different M3D core architectures. Finally, we undertake a full-system performance analysis with SPLASH-2 and PARSEC benchmarks.

\subsection{Experimental Setup}

In this work, we consider a 64-core two-tier M3D system where all cores are multi-tier. We use GEM5 [34], a full-system simulator, in FS mode running Linux to obtain detailed processor- and network-level information. We use the MOESI_CMP_directory cache coherency protocol with private $64 \mathrm{~KB} \mathrm{L1}$ instruction and data caches and a shared $8 \mathrm{MB}$ L2 cache. We consider SPLASH2 benchmark applications: FFT, RADIX, LU, WATER [35]; and PARSEC benchmark applications: CANNEAL (CAN) and FLUIDANIMATE (FLUID) [36]. The performance statistics generated by the GEM5 simulations are incorporated into McPAT to determine the power values [37].

For the DVFI strategy, we consider eight discrete V/F pairs listed in Table 2 for the different M3D architectures. The frequency values in the Iso configuration maintain a linear relationship 
Table 2. V/F Pairs for the Three M3D Systems

\begin{tabular}{c|c|c|c}
\hline Voltage level $(\mathrm{V})$ & Iso $(\mathrm{GHz})$ & Nä̈ve $(\mathrm{GHz})$ & Hetero $(\mathrm{GHz})$ \\
\hline 1.00 & 3.50 & 3.16 & 3.44 \\
\hline 0.95 & 3.20 & 2.90 & 3.16 \\
\hline 0.90 & 2.90 & 2.64 & 2.87 \\
\hline 0.85 & 2.58 & 2.35 & 2.56 \\
\hline 0.80 & 2.25 & 2.05 & 2.22 \\
\hline 0.75 & 1.90 & 1.73 & 1.88 \\
\hline 0.70 & 1.54 & 1.40 & 1.52 \\
\hline 0.65 & 1.18 & 1.07 & 1.17 \\
\hline
\end{tabular}

Table 3. VFI Clusters Size for Different Benchmarks

\begin{tabular}{l|c|c|c|c|c|r}
\hline & FFT & RADIX & WATER & CAN & FLUID & LU \\
\hline VFI1 & 29 & 37 & 33 & 22 & 40 & 32 \\
\hline VFI2 & 23 & 19 & 23 & 22 & 16 & 24 \\
\hline VFI3 & 7 & 4 & 4 & 16 & 4 & 4 \\
\hline VFI4 & 5 & 4 & 4 & 4 & 4 & 4 \\
\hline
\end{tabular}

following our discussion in Section 3. Similarly, the frequencies are scaled linearly for the Naïve and Hetero architectures for the eight voltage levels considered. On-chip voltage regulators allow fast transitions and low energy overheads [40]. The energy overhead of the voltage regulator and the DVFI controller is incorporated in the evaluation of the overall system. The IL-based DVFI controller was designed following Reference [30] in $28 \mathrm{~nm}$. The timing overhead of the IL-based DVFI controller is in the order of $\sim \mathrm{ns}$ (1.4 ns [30]), which is around $0.1 \%$ of each epoch interval considered in this work. The average energy consumed per decision is $0.044 \mathrm{~nJ}$ and the area overhead is only $0.022 \%$ of the total area of the system [30].

As discussed in Section 4.1, the hybrid clustering methodology from Reference [28] is used to create the four VFI clusters that maximize workload similarity within a VFI and minimize traffic between VFIs for an application. Table 3 shows the resulting cluster sizes for each benchmark. It should be noted that the number of VFIs depend on the tradeoff between hardware overhead and the performance constraints. In previous work [28], we have demonstrated that dividing 64 cores into four VFI clusters provides good energy efficiency without causing any noticeable performance penalty. Hence, in this work also we consider 64 cores divided into four VFIs. It should be noted that the size of VFI4 is always small compared to the other clusters. One possibility is to merge this cluster with other relatively bigger clusters. However, this directly affects the achievable V/F levels of the VFIs during runtime. Let us consider a simple example of the FFT benchmark on the Naïve M3D system, where we consider two clusters instead of four. The V/F levels of VFI4 are shown in Figure 5 when considered as a separate VFI and when merged with the nearest larger cluster (VFI2). The predicted voltage on the VFI4 cluster gets higher due to its shift towards a cluster with higher center value (i.e., higher IPC and traffic). Thus, EDP savings will reduce as the cores belonging to VFI4 run at a higher V/F value than always necessary.

\subsection{Imitation Learning for DVFI}

Following Section 4, we create the Oracle policy for each application. We allow the execution time penalty constraint to be $p=5 \%$ with respect to the corresponding no-VFI (NVFI) system while creating our DVFI learner policy. Each benchmark runtime is divided into around 1,000 epochs. The ACM Journal on Emerging Technologies in Computing Systems, Vol. 17, No. 2, Article 13. Pub. date: December 2020. 


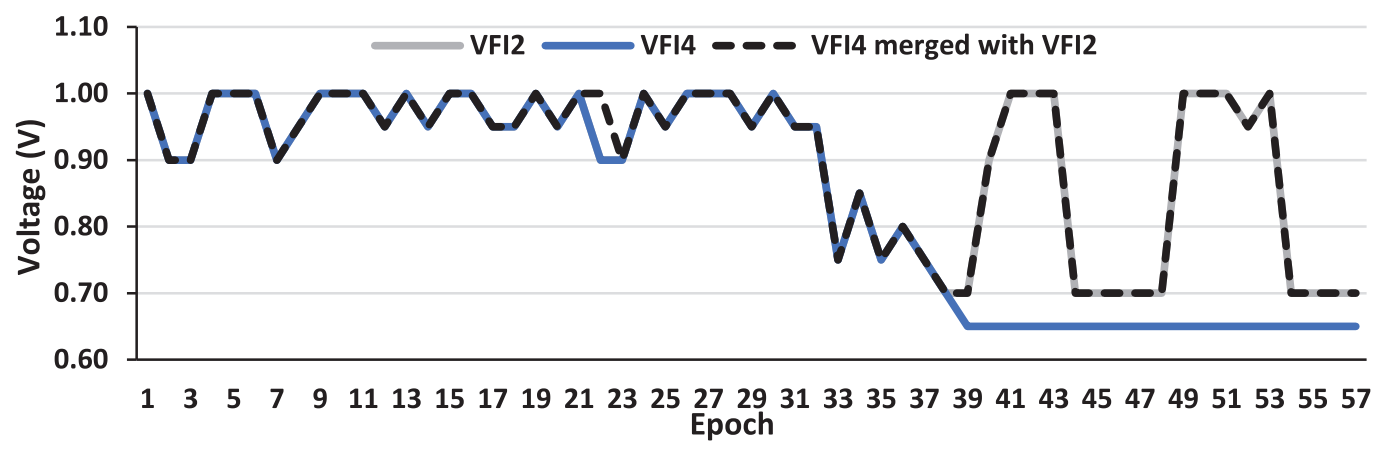

Fig. 5. Snapshot of selected voltage (applying DVFI explained in Section 5.2) for VFI2, VFI4, and VFI4 merged with VFI2 running the FFT benchmark.

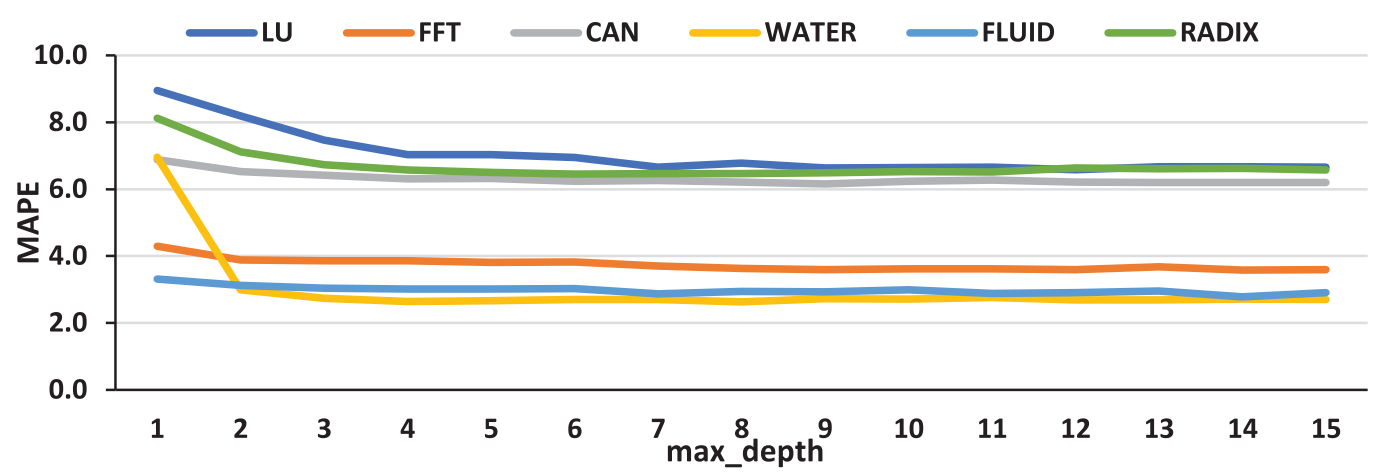

Fig. 6. The mean absolute percentage error (MAPE) rate of the random forest regressors with respect to the maximum tree depth (max_depth) considered for different benchmarks.

performance traces are collected per epoch (number of instructions, number of cycles, number of messages sent and received, etc.). Therefore, we have around 1,000 data samples for each benchmark in the Oracle set for the IL training process. First, we divide the Oracle set into a training set $(70 \%)$ and a testing set $(30 \%)$. We use IL to learn a random forest regression model (as discussed in Section 4.1 above). Random forest trains a collection of regression trees and uses the mean prediction of the individual trees to reduce overfitting. We have examined the error rate with respect to the maximum depth of the random forest regressors (max_depth) shown in Figure 6. The error curves saturate around max_depth $=8$. Therefore, we have considered the maximum depth of the random forest regressors to be 8 while learning our IL policies. We train a different regressor for each of the VFIs. Each regressor will use the VFI's current IPC, traffic, and V/F level as the input features and predict the appropriate V/F level for the VFI.

Since each M3D architecture has unique characteristics, we create different DVFI policies for each M3D architecture. We use the Iso architecture as the baseline, as it is the ideal system without any inter-tier process variation. Figure 7 shows VFI1's predicted output V/F levels, average core IPC, and average $f_{i j}$ over the runtime of the Naïve architecture running FFT as an example. The IPC and $f_{i j}$ are normalized to the range of $[0,1]$ in each case. Similarly, Figure 8 shows VFI1's predicted voltage level, average core IPC, and average $f_{i j}$ for the Hetero architecture running FFT. We observe that in both cases, the voltage levels predicted by the learner follows the variations in IPC and $f_{i j}$ : Higher values of IPC and $f_{i j}$ lead to a higher V/F level assignment while lower values result in lower $\mathrm{V} / \mathrm{F}$ levels. Although we see that the output voltage level follows the trend of the input IPC and 


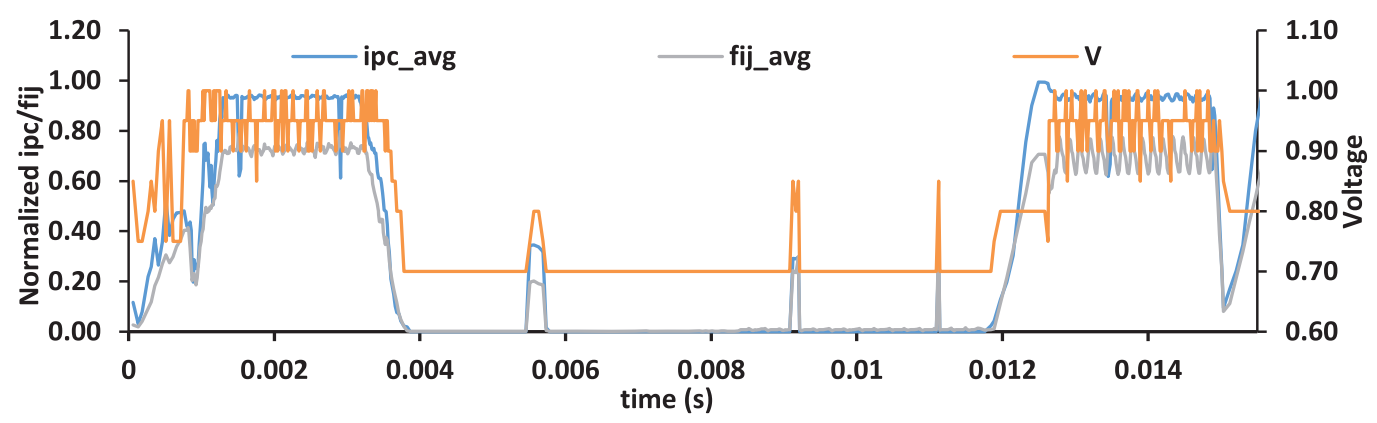

Fig. 7. VFI1's predicted output voltage (V), average ipc (ipc_avg), and average fij (fij_avg) over the runtime of the Naïve architecture running FFT.

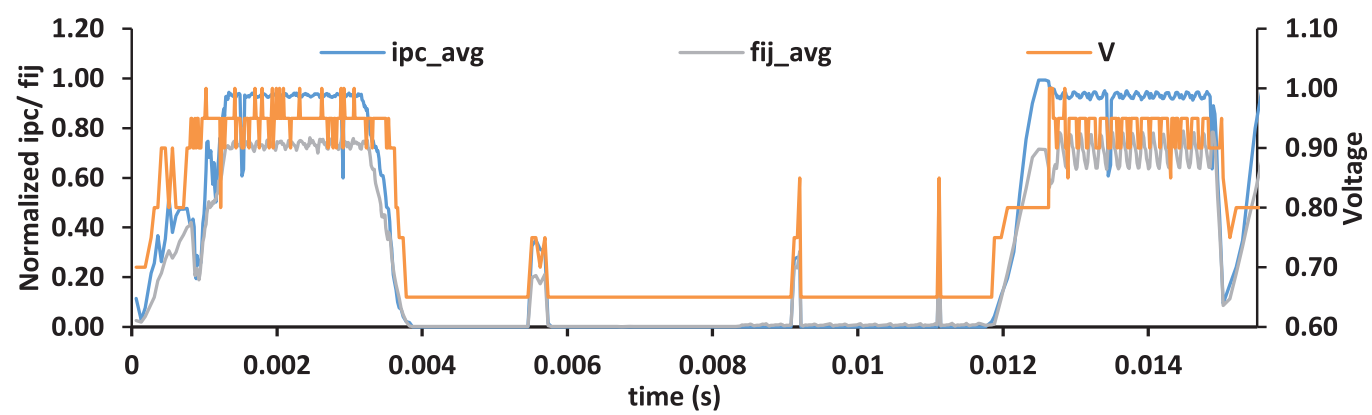

Fig. 8. VFI1's predicted output voltage (V), average ipc (ipc_avg), and average fij (fij_avg) over the runtime of the Hetero architecture running FFT.

traffic characteristics in a VFI for both Naïve and Hetero architectures, it is important to note that Hetero tends to have a lower value than the corresponding Naïve decision. This indicates that the mapping from system characteristics to output $\mathrm{V} / \mathrm{F}$ is different for the different M3D architectures.

To understand how the IL-policy is determining suitable control decisions based on the current state of the VFI, we show up to the third level of two example IL regression trees for Naïve (Figure 9(a)) and Hetero (Figure 9(b)) for the FFT benchmark. The input features considered are: \{average IPC (ipc_avg), maximum IPC (ipc_max), average $f_{i j}\left(\operatorname{tr} \_a v g\right)$, maximum $f_{i j}\left(\operatorname{tr} \_m a x\right)$, and previous window $\mathrm{V} / \mathrm{F}$ (prev_V/F)\}. Nodes with a lighter orange color indicate that the leaves of this subtree have lower $\mathrm{V} / \mathrm{F}$ levels and nodes with a darker orange coloring have higher $\mathrm{V} / \mathrm{F}$ levels. One important generalization that we can make for selecting V/F levels is that high IPC levels should use higher $\mathrm{V} / \mathrm{F}$ levels. The regression trees for the two architectures are different based on the process degradation effects on the IPC and communication characteristics of corresponding M3D systems.

The quality of the IL model can be assessed by how closely it follows the Oracle. This is evaluated using the mean absolute percentage error (MAPE) of the DVFI controller's V/F and the Oracle's $\mathrm{V} / \mathrm{F}$ for each application under consideration. Figure 10 shows the average MAPE (error bars show min and max) of the DVFI controllers across the four clusters in the system for both the Naïve and Hetero M3D architectures. We see a minimum value of $1.52 \%$ error in learning for WATER benchmark and a maximum of $8.24 \%$ error for LU benchmark. The average MAPE across all the benchmarks is $4.59 \%$. Thus, we see that the DVFI policy created for each M3D system architecture is able to robustly capture the application characteristics on the system and tune the V/F levels accordingly to minimize the EDP of the system. 


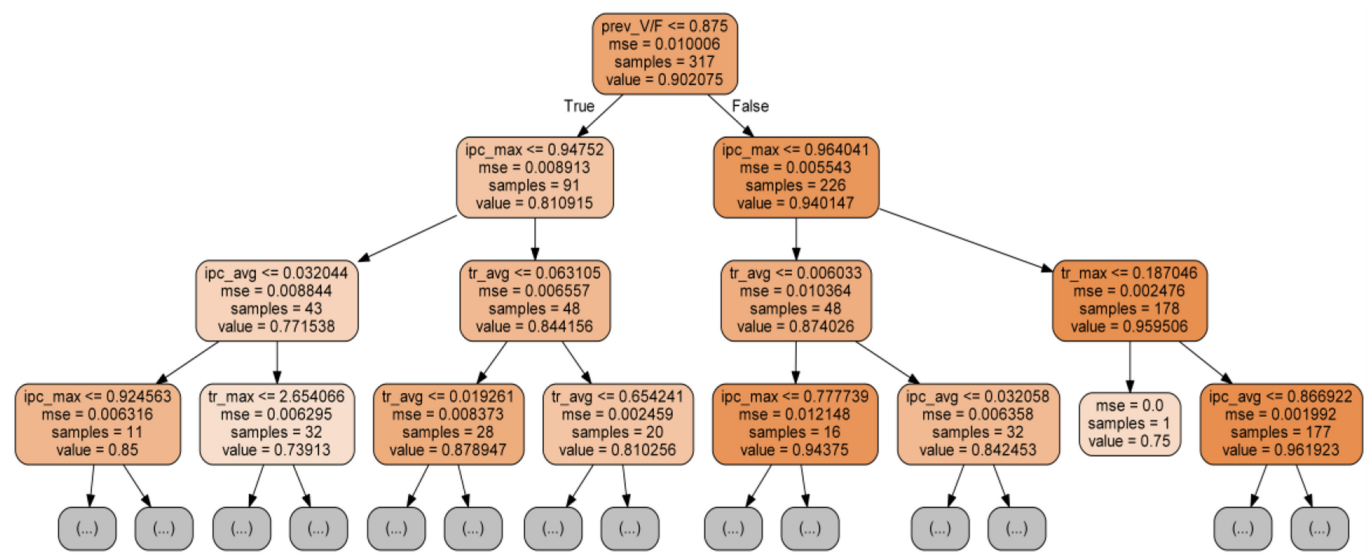

(a)
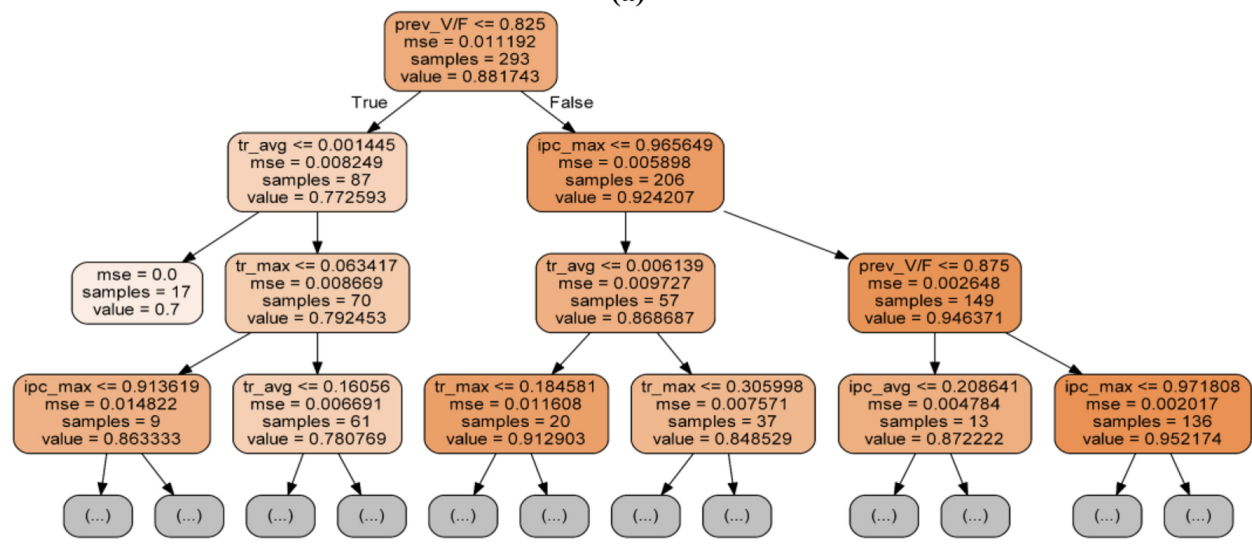

(b)

Fig. 9. Regression trees up to depth 3 for the FFT IL-policy on the (a) Naïve and (b) Hetero architecture (VFI1).

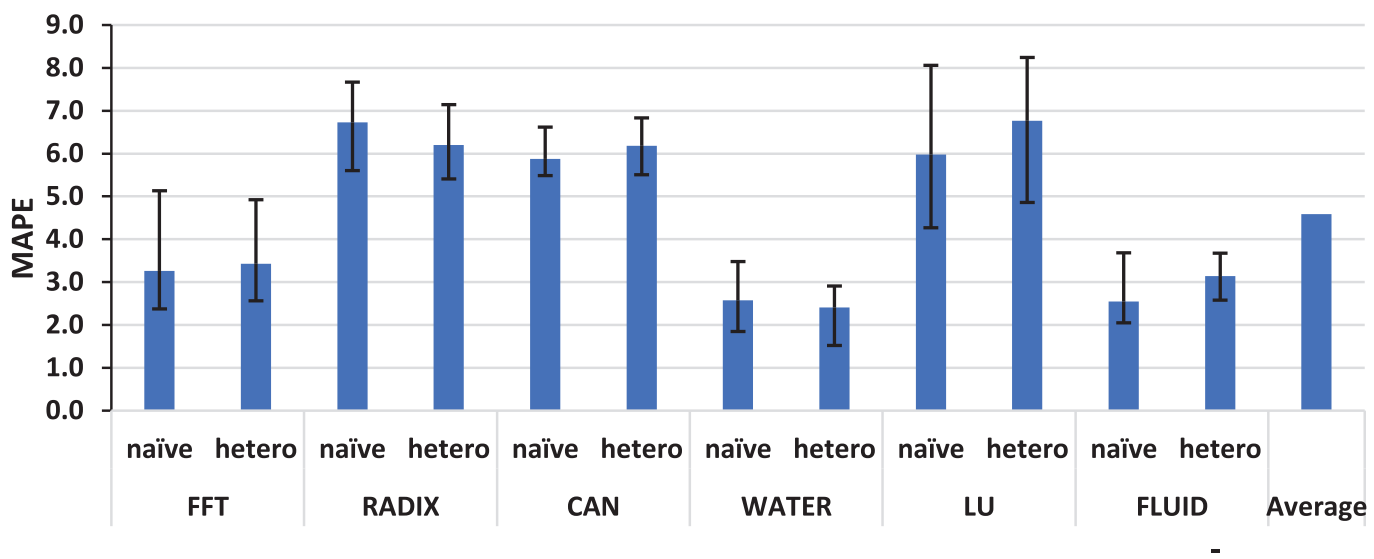

Fig. 10. Mean absolute percentage error (MAPE) between the predicted voltage of the IL-based DVFI controllers and the Oracle policies for each benchmark on the Naïve and Hetero M3D architectures. The error bars represent the minimum and maximum MAPE values across the VFI clusters on each system. 


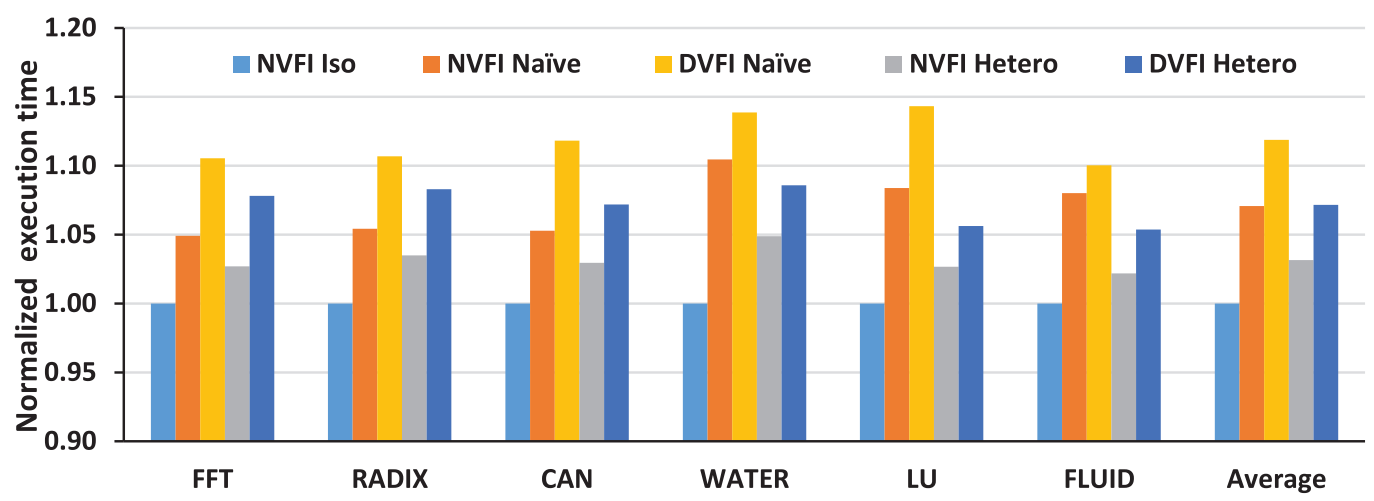

Fig. 11. Execution time of Nä̈ve and Hetero architectures for different benchmarks without any power management (NVFI) and with application of power-management policy (DVFI). The execution time values are normalized with respect to the Iso architecture.

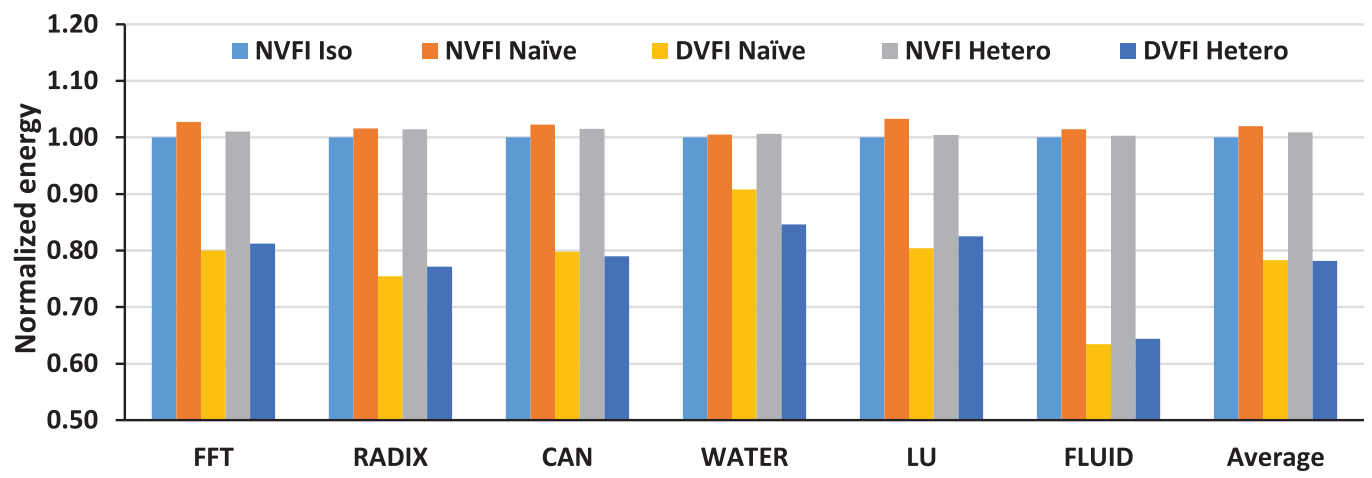

Fig. 12. Energy of Naïve and Hetero architectures for different benchmarks without any power management (NVFI) and with application of power-management policy (DVFI). The energy values are normalized with respect to the Iso architecture.

\subsection{Full-system Performance Evaluation}

We deploy the IL-based power-management strategy on the Naïve and Hetero architectures and compare their full-system execution time and energy dissipation. Here, we normalize the Naïve and Hetero performance with respect to Iso, which represents the ideal scenario. For each architecture, we also consider an NVFI system that runs at nominal V/F level without any power management. Figure 11 shows the execution time of the M3D systems with and without power management for each benchmark. The execution time is normalized with respect to the corresponding Iso system. The Naïve M3D system shows a significant slowdown due to the process variation effects. The Naïve NVFI system has an execution time penalty of $7 \%$ on average over the Iso system. Implementation of the DVFI policy increases the execution time penalty by $4.79 \%$ on average across all benchmarks. However, the Hetero architecture achieves faster execution due to its process-aware design. The execution time of NVFI Hetero, on average, is only $3.1 \%$ worse than that of the Iso architecture. DVFI Hetero architecture incurs an additional $4 \%$ execution time penalty on average. In summary, the DVFI Naïve and Hetero architectures incur $11.8 \%$ and $7.1 \%$ performance penalty, respectively, with respect to their Iso counterpart. Figure 12 shows the energy consumption of the different M3D systems. We achieve a significant energy improvement for the DVFI systems across 


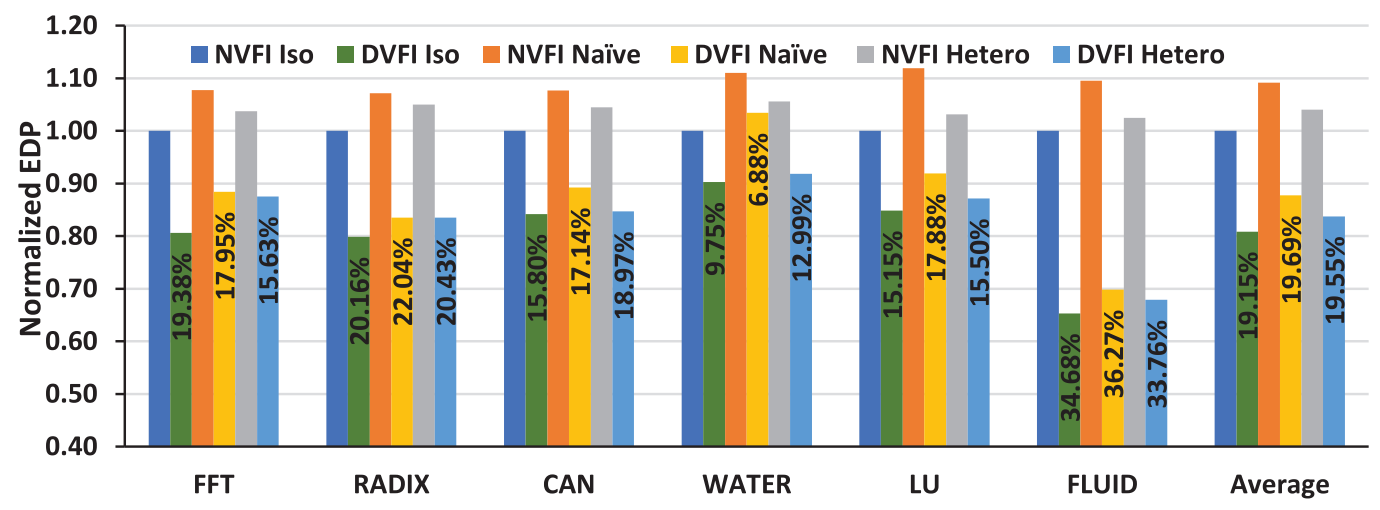

Fig. 13. EDP of Iso, Naïve, and Hetero architectures for different benchmarks without any power management (NVFI) and with application of power-management policy (DVFI). The EDP values are normalized with respect to the NVFI Iso architecture. The percentage levels on the DVFI bars indicate the improvement over the corresponding architecture's NVFI counterpart.

all the benchmarks. The average energy improvement for the Naïve M3D systems is $21.68 \%$ over the Iso architecture, while the energy improvement is $21.87 \%$ for the Hetero M3D systems across all the benchmark applications.

We see that Naïve architecture has good energy improvement for a small amount execution time penalty ( $4.79 \%$ on average over the NVFI Naïve; $11.8 \%$ on average with respect to Iso) even with all the process degradation in the system. The Hetero architecture is able to achieve lower execution time penalties ( $4 \%$ on average over NVFI Hetero; $7.1 \%$ on average with respect to Iso) with similar energy improvements as the design inherently considers the effects of inter-tier process variation.

The combined effects of execution time and energy consumption are captured in the unified performance metric of EDP. Figure 13 shows the EDP of all the M3D architectures with and without DVFI-based power management. The percentage levels on DVFI shown in Figure 13 indicate the improvement over the corresponding architecture's NVFI counterpart. On average, the DVFI Iso, Naïve, and Hetero architectures have $19.2 \%, 12.3 \%$, and $16.2 \%$ lower EDP, respectively, compared to the NVFI Iso architecture. It should be noted that DVFI Iso represents the ideal M3D scenario with no process variation and would naturally have the lowest EDP. These results demonstrate that our DVFI policy is able to learn good policies by considering the M3D process variation effects during runtime, even with different assumptions being made about M3D process variations during the architecture design stage.

\subsection{Process-aware vs. -unaware DVFI Policy}

In this section, we study the importance for considering the effects of process variation while implementing the DVFI policy for an M3D system. A process-unaware DVFI policy considers the M3D system to be ideal and learns ideal characteristics of the system (Iso DVFI policy). We will apply this Iso DVFI policy to the Naivve and Hetero systems to see how ignoring the process variation effects alter the results. Figures 14 and 15 show the performance degradation of the Naïve and Hetero architectures normalized to its NVFI system, respectively, when we apply the process-unaware Iso DVFI policy. It should be noted that when we created the Naïve-specific and Hetero-specific DVFI policies, our target was to maintain a performance penalty of $5 \%$ compared to NVFI. It is evident that both process-aware policies are able to maintain this performance penalty for most benchmarks with a few going slightly over that mark (maximum of $6.2 \%$ for CAN Naïve). However, we see a significant execution time penalty for deploying Iso policy on Nä̈ve architecture 


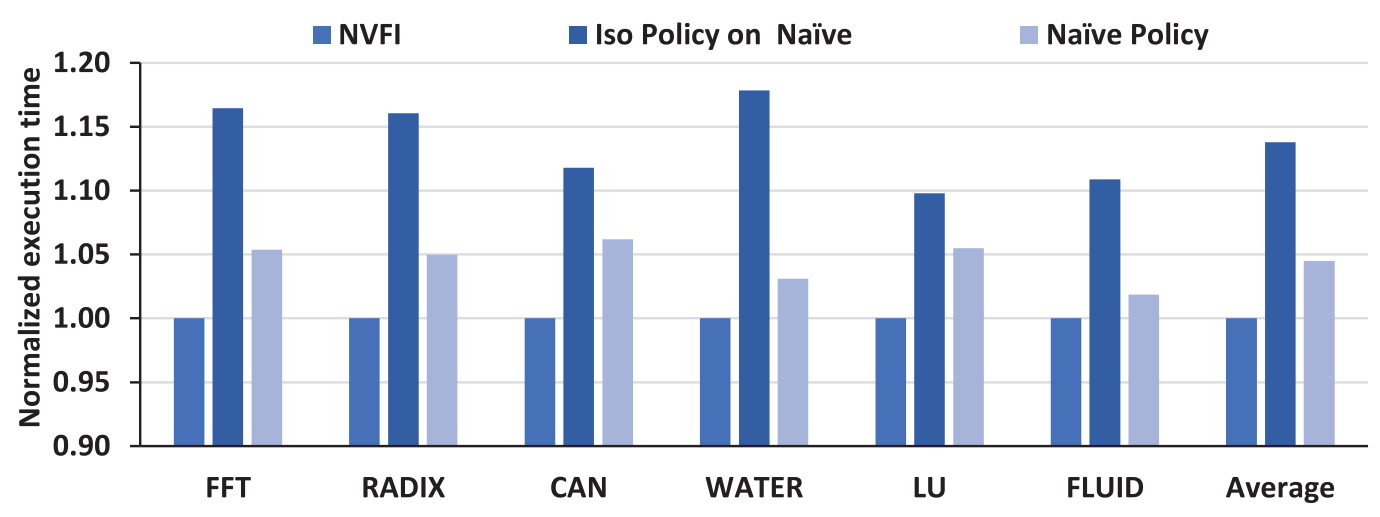

Fig. 14. The execution time of Naïve architecture applying the process-unaware Iso DVFI policy and its own DVFI policy with respect to the NVFI performance of Naïve architecture.

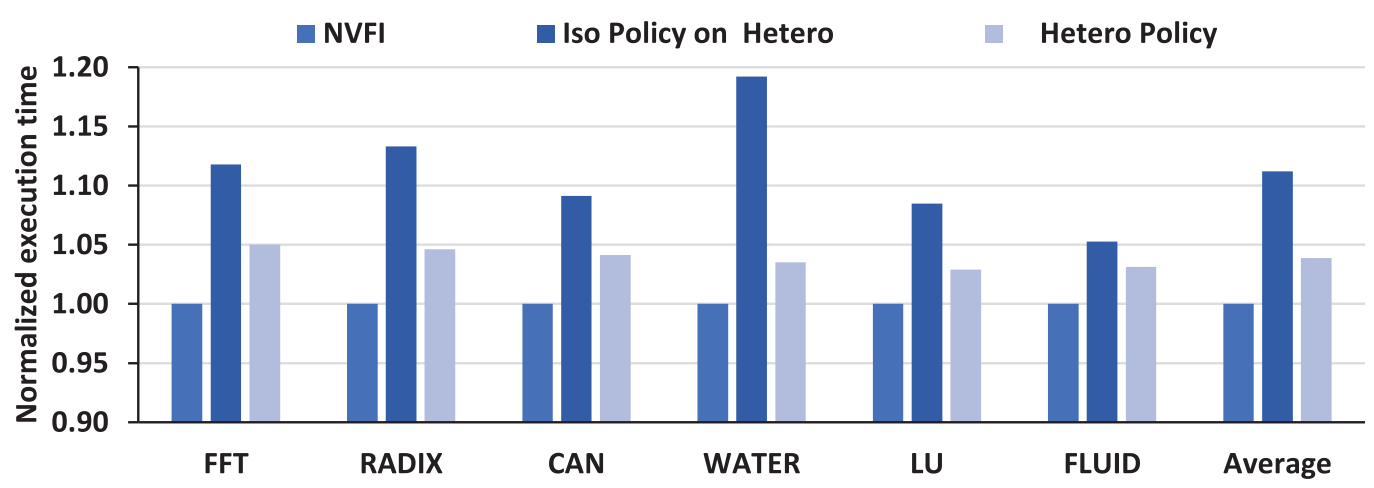

Fig. 15. The execution time of Hetero architecture applying the process-unaware Iso DVFI policy and its own DVFI policy with respect to the NVFI performance of Hetero architecture.

due to unaccounted process degradation effects while creating the DVFI policy. The performance degradation reaches up to $18 \%$ in case of WATER benchmark with an average increase in execution time of $13.8 \%$ across all the benchmarks compared to the Naïve NVFI. In the case of the Hetero architecture, we see the execution time penalty incurred on deploying the process-unaware Iso DVFI policy goes up to $19 \%$ (for WATER benchmark), with an average of $11.2 \%$ degradation across all benchmarks. The Iso DVFI policy on the Naïve and Hetero architectures are $8.9 \%$ and $7 \%$ worse, respectively, compared to the DVFI policies tuned to the specific process variations seen on each M3D system.

The above results demonstrate that process-unaware DVFI policies on M3D systems with intertier process variation violate the targeted performance constraints of the VFI system. A DVFI policy learned with the process degradation characteristics of the M3D systems incur less performance penalty (remaining within the target performance penalty). Therefore, power-management policies on M3D systems must consider the process variation effects.

\section{CONCLUSION}

Inter-tier process variation significantly affects the performance and energy efficiency of M3Dbased manycore chips. A VFI-based power-management strategy for M3D manycore systems should consider the impacts of inter-tier process variation to accurately determine the appropriate 
V/F levels to reduce energy consumption without significant performance overhead. An imitation learning (IL)-based DVFI implementation learns the M3D system characteristics in presence of process variation and allows dynamic $\mathrm{V} / \mathrm{F}$ tuning to design an energy-efficient manycore system. We designed a robust IL-based power-management policy that works equally well for both processoblivious and process-aware core architectures. On average, the process-variation-aware IL-based DVFI policy lowers the overall EDP of the process-aware Hetero system by $16.2 \%$ and the processoblivious Naïve system by $12.3 \%$ with respect to the EDP of an ideal M3D system, Iso across all the benchmarks considered in this work. Most importantly, we demonstrate that not considering the effects of process variation while designing the power-management policy of an M3D core chip results in very optimistic $\mathrm{V} / \mathrm{F}$ selections and performance degradations that significantly violate target performance constraints.

\section{REFERENCES}

[1] W. R. Davis, J. Wilson, S. Mick, J. Xu, H. Hua, C. Mineo, A. M. Sule, M. Steer, and P. D. Franzon. 2005. Demystifying 3D ICs: The pros and cons of going vertical. IEEE Des. Test Comput. 22, 6 (2005), 498-510.

[2] K. Sakuma, P. S. Andry, C. K. Tsang, S. L. Wright, B. Dang, C. S. Patel, B. C. Webb, J. Maria, E. J. Sprogis, S. K. Kang, and R. J. Polastre. 2008. 3D chip-stacking technology with through-silicon vias and low-volume lead-free interconnections. IBM 7. Res. Dev. 52, 6 (2008), 611-622.

[3] G. Van der Plas, P. Limaye, I. Loi, A. Mercha, H. Oprins, C. Torregiani, S. Thijs, D. Linten, M. Stucchi, G. Katti, and D. Velenis. 2010. Design issues and considerations for low-cost 3-D TSV IC technology. IEEE f. Solid-State Circ. 46, 1 (2010), 293-307.

[4] H. H. S. Lee and K. Chakrabarty. 2009. Test challenges for 3D integrated circuits. IEEE Des. Test Comput. 26, 5 (2009), $26-35$.

[5] Y. J. Lee, D. Limbrick, and S. K. Lim. 2013. Power benefit study for ultra-high density transistor-level monolithic 3D ICs. In Proceedings of the Design Automation Conference (DAC'13). 1-10.

[6] P. Batude, B. Sklenard, C. Fenouillet-Beranger, B. Previtali, C. Tabone, O. Rozeau, O. Billoint, O. Turkyilmaz, H. Sarhan, S. Thuries, G. Cibrario, L. Brunet, F. Deprat, J.-E. Michallet, F. Clermidy, and M. Vinet. 2014. 3D sequential integration opportunities and technology optimization. In Proceedings of the IEEE International Interconnect Technology Conference (IITC'14). 373-376.

[7] D. K. Nayak, S. Banna, S. K. Samal, and S. K. Lim. 2015. Power, performance, and cost comparisons of monolithic 3D ICs and TSV-based 3D ICs. In Proceedings of the IEEE SOI-3D-Subthreshold Microelectronics Technology Unified Conference (S3S'15). 1-2.

[8] O. Turkyilmaz, G. Cibrario, O. Rozeau, P. Batude, and F. Clermidy. 2014. 3D FPGA using high-density interconnect monolithic integration. In Proceedings of the Conference on Design, Automation \& Test in Europe (DATE'14). 1-4.

[9] S. Das, J. R. Doppa, P. P. Pande, and K. Chakrabarty. 2017. Monolithic 3D-enabled high performance and energy efficient network-on-chip. In Proceedings of the IEEE International Conference on Computer Design (ICCD'17). 233224.

[10] B. Rajendran, R. S. Shenoy, D. J. Witte, N. S. Chokshi, R. L. DeLeon, G. S. Tompa, and R. F. W. Pease. 2007. Low thermal budget processing for sequential 3-D IC fabrication. IEEE Trans. Electron Dev. 54, 4 (2007), 707-714.

[11] P. Batude, T. Ernst, J. Arcamone, G. Arndt, P. Coudrain, and P. Gaillardon. 2012. 3-D sequential integration: A key enabling technology for heterogeneous co-integration of new function with CMOS. IEEE F. Emerg. Select. Top. Circ. Syst. 2, 4 (2012), 714-722.

[12] S. Musavvir, A. Chatterjee, R. G. Kim, D. H. Kim, and P. P. Pande. 2020. Inter-tier process-variation-aware monolithic 3-D NoC design space exploration. IEEE Trans. Very Large Scale Integ. (VLSI) Syst. 28, 3 (2020), 686-699.

[13] D. Lee, S. Das, J. R. Doppa, P. P. Pande, and K. Chakrabarty. 2018. Performance and thermal tradeoffs for energyefficient monolithic 3D network-on-chip. ACM Trans. Des. Autom. Electron. Syst. 23, 5 (2018), 1-25.

[14] S. Bobba, A. Chakraborty, O. Thomas, P. Batude, and G. de Micheli. 2013. Cell transformations and physical design techniques for 3D monolithic integrated circuits. F. Emerg. Technol. Comput. Syst. 9, 3 (2013), 1-28.

[15] M. Lin, A. El Gamal, Y. Lu, and S. Wong. 2007. Performance benefits of monolithically stacked 3-D FPGA. IEEE Trans. Comput.-Aided Des. Integ. Circ. Syst. 26, 2 (2007), 216-229.

[16] T. Uhrmann, T. Wagenleitner, T. Glinsner, M. Wimplinger, and P. Lindner. 2014. Monolithic IC integration key alignment aspects for high process yield. In Proceedings of the SOI-3D-Subthreshold Microelectronics Technology Unified Conference (S3S'14). 1-2.

[17] Y. Lee and S. K. Lim. 2013. Ultrahigh density logic designs using monolithic 3-D integration. IEEE Trans. Comput.Aided Des. Integ. Circ. Syst. 32, 12 (2013), 1892-1905. 
[18] Y. J. Lee, P. Morrow, and S. K. Lim. 2012. Ultra high density logic designs using transistor-level monolithic 3D integration. In Proceedings of the IEEE/ACM International Conference on Computer-Aided Design (ICCAD'12). 539-546.

[19] S. K. Samal, D. Nayak, M. Ichihashi, S. Banna, and S. K. Lim. 2016. Monolithic 3D IC vs. TSV-based 3D IC in 14nm FinFET technology. In Proceedings of the IEEE SOI-3D-Subthreshold Microelectronic Technology Unified Conference (S3S'16). 1-2.

[20] K. Acharya, K. Chang, B. W. Ku, S. Panth, S. Sinha, B. Cline, G. Yeric, and S. K. Lim. 2016. Monolithic 3D IC design: Power, performance, and area impact at $7 \mathrm{~nm}$. In Proceedings of the 17th International Symposium on Quality Electronic Design (ISQED'16). 41-48.

[21] K. M. Kim, S. Sinha, B. Cline, G. Yeric, and S. K. Lim. 2016. Four-tier monolithic 3D ICs: Tier partitioning methodology and power benefit study. In Proceedings of the International Symposium on Low Power Electronics and Design. 70-75.

[22] S. Panth, S. K. Samal, K. Samadi, Y. Du, and S. K. Lim. 2017. Tier degradation of monolithic 3-D ICs: A power performance study at different technology nodes. IEEE Trans. Comput.-Aided Des. Integ. Circ. Syst. 36, 8 (2017), 1265-1273.

[23] L. Pasini, P. Batude, M. Cassé, L. Brunet, P. Rivallin, B. Mathieu, J. Lacord, S. Martinie, C. Fenouillet-Beranger, B. Previtali, N. Rambal, M. Haond, G. Ghibaudo, and M. Vinet. 2014. nFET FDSOI activated by low temperature solid phase epitaxial regrowth: Optimization guidelines. In Proceedings of the SOI-3D-Subthreshold Microelectronics Technology Unified Conference (S3S'14). 1-2.

[24] C. Fenouillet-Beranger, B. Mathieu, B. Previtali, M. Samson, N. Rambal, V. Benevent, S. Kerdiles, J. Barnes, D. Barge, P. Besson, R. Kachtouli, M. Cassé, X. Garros, A. Laurent, F. Nemouchi, K. Huet, I. Toqué-Trésonne, D. Lafond, H. Dansas, F. Aussenac, G. Druais, P. Perreau, E. Richard, S. Chhun, E. Petitprez, N. Guillot, F. Deprat, L. Pasini, L. Brunet, V. Lu, C. Reita, P. Batude, and M. Vinet. 2014. New insights on bottom layer thermal stability and laser annealing promises for high performance 3D VLSI. In Proceedings of the IEEE International Electron Device Meeting. 27, 5 (2014) 1-27.

[25] B. Gopireddy and J. Torrellas. 2019. Designing vertical processors in monolithic 3D. In Proceedings of the 46th International Symposium on Computer Architecture (ISCA'19). Association for Computing Machinery, 643-656.

[26] U. Y. Ogras, R. Marculescu, D. Marculescu, and E. G. Jung. 2009. Design and management of voltage-frequency island partitioned networks-on-chip. IEEE Trans. Very Large Scale Integ. Circ. Syst. 17 (2009), 330-341.

[27] D. C. Juan, S. Garg, J. Park, and D. Marculescu. 2013. Learning the optimal operating point for many-core systems with extended range voltage/frequency scaling. In Proceedings of the IEEE/ACM/IFIP International Conference on Hardware/Software Codesign and System Synthesis (CODES+ISSS'13). 1-10.

[28] R. G. Kim, W. Choi, Z. Chen, P. P. Pande, D. Marculescu, and R. Marculescu. 2016. Wireless NoC and Dynamic VFI codesign: Energy efficiency without performance penalty. IEEE Trans. Very Large Scale Integ. (VLSI) Syst. 24, 7 (2016), 2488-2501.

[29] C. Ababei and M. G. Moghaddam. 2019. A survey of prediction and classification techniques in multicore processor systems. IEEE Trans. Parallel Distrib. Syst. 30, 5 (2019), 1184-1200.

[30] R. G. Kim, W. Choi, Z. Chen, J. R. Doppa, P. P. Pande, D. Marculescu, and R. Marculescu. 2017. Imitation learning for dynamic VFI control in large-scale manycore systems. IEEE Trans. Very Large Scale Integ. (VLSI) Syst. 25, 9 (2017), 2458-2471.

[31] M. M. Shulaker, T. F. Wu, M. M. Sabry, H. Wei, H. P. Wong, and S. Mitra. 2015. Monolithic 3D integration: A path from concept to reality. In Proceedings of the Design, Automation \& Test in Europe Conference \& Exhibition (DATE'15). $1197-1202$.

[32] K. Athikulwongse, A. Chakraborty, J.-S. Yang, D. Z. Pan, and S. K. Lim. 2010. Stress-driven 3D-IC placement with TSV keep-out zone and regularity study. In Proceedings of the IEEE/ACM International Conference on Computer-Aided Design (ICCAD’10). 669-674.

[33] S. Ross, G. J. Gordon, and J. A. Bagnell. 2011. A reduction of imitation learning and structured prediction to no-regret online learning. In Proceedings of the International Conference on Artificial Intelligence and Statististics. 627-635.

[34] N. Binkert, B. Beckmann, G. Black, S. K. Reinhardt, A. Saidi, A. Basu, J. Hestness, D. R. Hower, T. Krishna, S. Sardashti, R. Sen, K. Sewell, M. Shoaib, N. Vaish, M. D. Hill, and D. A. Wood. 2011. The gem5 simulator. SIGARCH Comput. Archit. News 39, 2 (2011), 1-7.

[35] S. C. Woo, M. Ohara, E. Torrie, J. P. Singh, and A. Gupta. 1995. The SPLASH-2 programs: Characterization and methodological considerations. In Proceedings of the International Symposium on Computer Architecture (ISCA'95). 24-36.

[36] C. Bienia. 2011. Benchmarking Modern Multiprocessors. Ph.D. Dissertation. Princeton University, Princeton, NJ.

[37] S. Li, J. H. Ahn, R. D. Strong, J. B. Brockman, D. M. Tullsen, and N. P. Jouppi. 2009. McPAT: An integrated power, area, and timing modeling framework for multicore and manycore architectures. In Proceedings of the IEEE/ACM International Symposium on Microarchitecture (MICRO'09). 469-480.

[38] S. Das, J. R. Doppa, P. P. Pande, and K. Chakrabarty. 2017. Robust TSV-based 3D NoC design to counteract electromigration and crosstalk noise. In Proceedings of the Design, Automation and Test in Europe Conference (DATE'17). 1366-1371. 
[39] S. Das, J. R. Doppa, P. P. Pande, and K. Chakrabarty. 2017. Design space exploration and optimization of an energyefficient and reliable 3-D small-world network-on-chip. IEEE Trans. Comput.-Aided Des. Integ. Circ. Syst. 36, 5 (2017), $719-732$.

[40] J. Murray, N. Tang, P. P. Pande, D. Heo, and B. A. Shirazi. 2015. DVFS pruning for wireless NoC architectures. IEEE Trans. Des. Test 32, 2 (2015), 29-38.

[41] S. Borkar. 2011. 3D integration for energy efficient system design. In Proceedings of the 48th Design Automation Conference (DAC'11). 214-219.

[42] ITRS Reports. 2015. The International Technology Roadmap for Semiconductors. Retrieved from http://www.itrs2. net/itrs-reports.html.

[43] F. Li, C. Nicopoulos, T. Richardson, Y. Xie, V. Narayanan, and M. Kandemir. 2006. Design and management of 3D chip multiprocessors using network-in-memory. In Proceedings of the IEEE International Symposium on Computer Architecture (ISCA'06). 130-141.

[44] U. Kang, H. J. Chung, S. Heo, S. H. Ahn, H. Lee, S. H. Cha, J. Ahn, D. Kwon, J. H. Kim, J. W. Lee, H. S. Joo, W. S. Kim, H. K. Kim, E. M. Lee, S. R. Kim, K. H. Ma, D. H. Jang, N. S. Kim, M. S. Choi, S. J. Oh, J. B. Lee, T. K. Jung, J. H. Yoo, and C. Kim. 2009. 8Gb 3D DDR3 DRAM using through-silicon-via technology. In Proceedings of the IEEE International Solid-State Circuits Conference Digest of Technical Papers. 130-131.

[45] M. Koyanagi, T. Nakamura, Y. Yamada, H. Kikuchi, T. Fukushima, T. Tanaka, and H. Kurino. 2006. Three-dimensional integration technology based on wafer bonding with vertical buried interconnections. IEEE Trans. Electron Dev. 53 , 11 (2006), 2799-2808.

[46] A. Rahmani, M. Haghbayan, A. Kanduri, A. Y. Weldezion, P. Liljeberg, J. Plosila, A. Jantsch, and H. Tenhunen. 2015. Dynamic power management for many-core platforms in the dark silicon era: A multi-objective control approach. In Proceedings of the IEEE/ACM International Symposium on Low Power Electronics and Design (ISLPED'15). 219-224.

[47] W. Sun, A. Venkatraman, G. J. Gordon, B. Boots, and J. A. Bagnell. 2017. Deeply AggreVaTeD: Differentiable imitation learning for sequential prediction. In Proceedings of the 34th International Conference Machine Learning 70 (2017), 3309-3318.

Received May 2020; revised August 2020; accepted October 2020 